\title{
Mesenchymal Stem/Stromal Cells Exert Trophic Effect on Colorectal Cancer Metastasis to the Liver
}

\author{
Pratika Y Hernanda ${ }^{1}$, Alexander Pedroza-Gonzalez ${ }^{1}$, Luc J W van der Laan ${ }^{2}$, Martin J Hoogduijn ${ }^{3}$, Maikel P Peppelenbosch ${ }^{1}$ and Qiuwei \\ Pan $^{1 *}$
}

${ }^{1}$ Department of Gastroenterology and Hepatology, Erasmus University Medical Center, Rotterdam, 3015 CE, The Netherlands

${ }^{2}$ Department of Surgery, Erasmus University Medical Center, Rotterdam, 3015 CE, The Netherlands

${ }^{3}$ Department of Internal Medicine, Erasmus University Medical Center, Rotterdam, 3015 CE, The Netherlands

\begin{abstract}
Colorectal Cancer (CRC) is the third most common cancer in the world. CRC tends to metastasize to the liver, which may occur in $20 \%$ to $70 \%$ of patients and represents the major cause of death. Mesenchymal Stem/stromal cells (MSCs) have shown to be able to migrate to CRC site and play an important role in tumor progression. We have previously identified a resident MSC population in the liver. Therefore, this study aims to investigate whether there is infiltration of MSCs into patient CRC Liver Metastasis (CRC-LM) and their potential effects on tumor cell growth. By culturing resected patient CRC-LM tissue, we observed the emerging of fibroblast-like cells. Further phenotype and functional characterization confirmed their bonafide MSCs features. In situ staining with a well-established MSCs marker showed a significant enrichment of candidate MSCs in patient CRC-LM, particularly the tumor-stromal area. Moreover, MSCs secreted trophic factors significantly increased colony formation and growth of a metastatic CRC cell line. In summary, we found infiltration and enrichment of MSCs in CRC-LM patient, which could in turn nourish tumor cells.
\end{abstract}

Keywords: Mesenchymal stem/stromal cells; Colorectal liver metastasis; Trophic action; Patients

\section{Introduction}

Colorectal Cancer (CRC) is the third most common cancer in the world. An estimated 1.24 million people worldwide were diagnosed with colorectal cancer in 2008 , accounting for $10 \%$ of the total cancer patients (http://globocan.iarc.fr/ ). In approximately $75-80 \%$ of cases, patients have potentially resectable disease at the time of diagnosis [1]. Liver confined metastases (synchronous metastases) are found in $77 \%$ of CRC patients presenting stage IV disease at diagnosis [2]. The treatment of CRC has evolved greatly in the last 10 years, involving complex combined chemotherapy protocols and, in more recent times, new biologic agents. Evidence to date suggests potentially distinct roles for bevacizumab and EGF receptor-targeted biological agents (cetuximab and panitumumab) in the treatment of metastatic CRC. Advances in adjuvant therapy have been limited to the addition of oxaliplatin and the substitution of oral fluoropyrimidine (e.g., capecitabine) with intravenous 5-fluorouracil, however there was no evidence for improved outcome with biological agents [3]. Therefore, new therapeutic options are urgently needed for advanced or metastatic CRC.

Mesenchymal Stem/stromal Cells (MSCs) were first found in the Bone Marrow (BM) that act as stromal cells supporting hematopoietic stem cells [4]. In recent years, MSCs have been extensively demonstrated possessing multilineage differentiation potential, anti-inflammatory function and immunomodulatory properties [5]. More recently, the tumor-trophic and migratory properties of MSCs demonstrated in various pre-clinical models has emerged an interesting concept of using MSCs as carrier for anti-cancer drug/gene delivery [6,7]. This notion also appears to be supported by several studies showing antitumor effects of MSCs [8-10]. In contrast, others reported that MSCs could promote tumor progression or metastasis in animal models [1113]. Thus, it is still unclear, regarding the pro- or anti-cancer role of MSCs, in particular the effects in patients [14].
We have previously identified a resident MSC population within the human adult liver that are phenol typically and functionally similar to BM MSCs [15]. We subsequently demonstrated that MSCs are enriched in human primary liver cancer (hepatocellular carcinoma; HCC) and could exert trophic effects on tumor cells [16]. This prompted us to further investigate the presence and the roles of MSCs in liver metastatic CRC (CRC-LM).

\section{Materials and Methods}

\section{Patient samples}

Nine paired fresh CRC-LM and tumor-free liver tissue were collected for MSCs culturing. 12 paraffin-embedded patient CRC-LM tissues were collected at the tissue bank at the Erasmus MC Rotterdam for immunohistochemical staining of MSC marker (Table 1). The medical ethical committee of Erasmus MC proved the use of patient materials.

\section{Procedure of culturing MSCs}

Adjacent liver tissue or CRC-LM tumor were digested into single cell suspensions or cut into small pieces. Single cell suspension or small pieces of tissues were cultured for MSCs as previously described [16].

*Corresponding author: Quuwei Pan, Department of Gastroenterology and Hepatology, Erasmus University Medical Center, Rotterdam, The Netherlands, Tel: +31-10-703-7502; Fax: +31-10-703-2793; E-mail: q.pan@erasmusmc.nl

Received October 30, 2013; Accepted November 14, 2013; Published November 21, 2013

Citation: Hernanda PY, Gonzalez AP, van der Laan LJW, Hoogduijn MJ Peppelenbosch MP, et al. (2013) Mesenchymal Stem/Stromal Cells Exer Trophic Effect on Colorectal Cancer Metastasis to the Liver. J Liver 2: 135. doi:10.4172/2167-0889.1000135

Copyright: (c) 2013 Hernanda PY, et al. This is an open-access article distributed under the terms of the Creative Commons Attribution License, which permits unrestricted use, distribution, and reproduction in any medium, provided the original author and source are credited. 


\begin{tabular}{|c|c|c|c|c|}
\hline Patient No. & Gender & Recurrence & Cirrhosis & Hepatitis \\
\hline $\mathbf{1}$ & M & yes & no & no \\
\hline $\mathbf{2}$ & M & yes & no & no \\
\hline $\mathbf{3}$ & M & yes & no & no \\
\hline $\mathbf{4}$ & F & yes & no & no \\
\hline $\mathbf{5}$ & F & no & no & no \\
\hline $\mathbf{6}$ & F & no & no & no \\
\hline $\mathbf{7}$ & M & no & no & no \\
\hline $\mathbf{8}$ & M & yes & no & no \\
\hline $\mathbf{9}$ & M & yes & no & no \\
\hline $\mathbf{1 0}$ & M & no & no & no \\
\hline $\mathbf{1 1}$ & F & no & no & no \\
\hline $\mathbf{1 2}$ & M & no & no & no \\
\hline & Table 1: Characteristics of CRC-LM patients. & \\
\hline
\end{tabular}

\section{Flow cytometric analysis}

Cells were stained with mouse monoclonal antibodies against CD13-PECy7 (BD Biosciences), CD34-APC (BD Biosciences), CD45PERCP (BD Biosciences), CD73-PE (BD Pharma, San Jose, CA), CD105-FITC (R\&D Systems, Abingdon, UK), CD166-PE (BD Pharma, San Jose, CA) and HLA-I-APC(BD Biosciences), and flow cytometric analysis was performed.

\section{Assay of adipogenic and osteogenic differentiation}

Adipogenic andosteogenic differentiation were performed as previously described [16]. Oil Red O staining was used for adipocytes detection. Alizarin Red S staining was used to detect deposited calcium phosphates.

\section{Colony forming assay of SW620 cells}

The colony formation assay was performed in SW620 cells, the metastasis colorectal cancer cell lines [17], 1000 cells were plated in each well of 6 wells plate and were treated with MSCs conditioned medium (MSC-CM), which was prepared by culturing MSCs until $70-90 \%$ confluence and medium was collected 48 hours post-culture. Colony formation assay was also performed with SW620 cells only in DMEM medium with $10 \%$ fetal bovine serum with or without MSCCM. SW620 colonies were stained with haematoxylin \& eosin after two weeks.

\section{Immunohistochemistry staining}

Paraffin embedded CRC-LM tumor tissue slides were used to stain with the mouse monoclonal antibody STRO-1 (Invitrogen Corporation) and then counterstained with haematoxylin [16].

\section{Statistical analysis}

Statistical analysis was performed by using the Graph Pad Software with nonparametric Mann-Whitney test or paired t-test. P-values $<0.05$ were considered statistically significant.

\section{Results}

\section{Culture of MSCs from resected human CRC-LM tissues}

To investigate whether MSCs are present in patient CRC-LM tumors, surgical resection of CRC-LM tissues of 9 patients were collected and submitted to an MSC culturing protocol previous validated for liver tissue $[14,16]$. Indeed, mesenchymal like cells were easily identified in the resulting cultures (Figure 1A). Upon subculturing, rapid grow out and expansion of this cell type was validated (Figure 1B). To confirm the bona fide MSCs nature of these cells, a functional analysis was performed and demonstrated that these tumor-derived cells had a multilineage potential with a capacity for adipogenic (Figure 1C) and osteogenic differentiation (Figure 1D). In addition, FACS analysis confirmed that these cells are positive for common mesenchymal markers CD13, CD73, CD105 and CD166 and are negative for the common hematopoietic markers CD34 and CD45 (Figure 2A). Finally, STRO-1 is considered as the well-known MSCs marker [18], in particular for in vivo immunohistochemical staining of candidate MSCs [7]. The STRO-1 protein is highly expressed in MSCs cultured from CRC-LM tissues, as evident from immunohistochemical staining (Figure 2B).

\section{STRO-1 Cells are enriched in human CRC-LM tumors}

To further characterize whether the presence of MSCs is a general phenomenon in CRC metastasis to the liver, we investigated a further cohort of patients using paraffin-embedded patient CRC-LM ( $\mathrm{n}=12$, Table 1) and immunohistochemical staining of STRO-1. STRO-1 positive cells were detected in both normal adjacent tissue and tumor areas (Figure $3 \mathrm{~A})$. In the regions of the liver apparently unaffected by the cancerous process, STRO-1 positive cells mainly locate in liver sinusoid or blood veins. Interestingly, the frequency of STRO-1 in tumor, in particular the tumor stromal region, is significantly higher than normal adjacent tissue site (Figure 3B). Thus the presence of MSCs is not only a characteristic of CRC metastasis to the liver, there is also active recruitment of MSCs to the tumor and these cells are locally enriched in the metastasis. MSCs are also present in the primary CRC tumor but hardly detectable in the normal adjacent colon tissue (Figure 3C).

\section{MSCs secret trophic factors that can enhance colony unit formation and growth of human metastatic CRC cells}

Previously, we showed that human liver MSCs can exert trophic action on HCC cells [16], but whether these cells have the capacity to foster CRC cell is unknown. Thus we decided to investigate whether factors secreted by MSCs influence colony unit formation and growth of the SW620 CRC cell line. This cell line was initially isolated from a
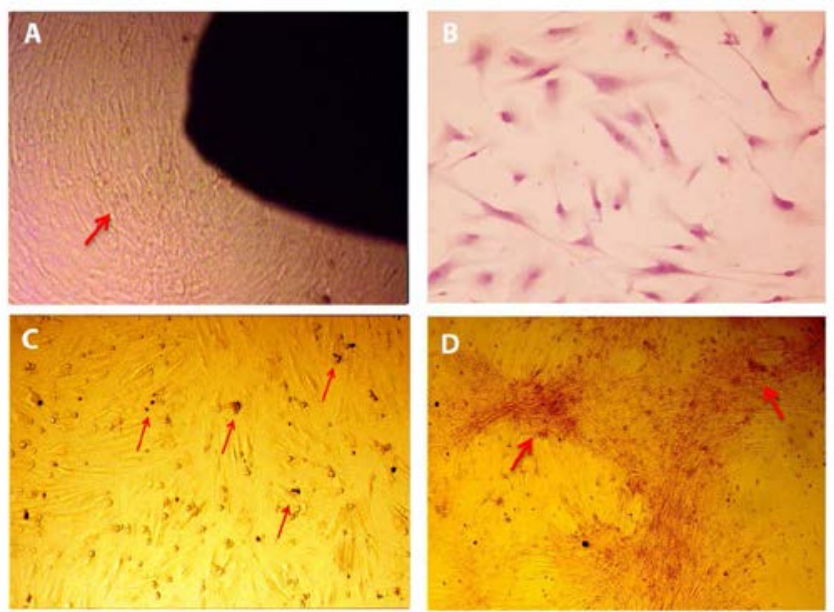

Figure 1: (A) Culture and lineage differentiation of MSCs from human CRC-LM tissues. With a method of culturing tiny tissue specimen from surgical resected human CRC-LM tissues, fibroblast-like cells appeared. (B) These cells could rapidly grow out and expand by subculture showing typical MSCs morphology. (C) Adipogenic differentiation of CRC-LM derived MSCs, detected by Oil red O staining for lipid droplet (Arrow). (D) Osteogenic differentiation of these cells was evaluated by detection of deposited calcium phosphates using Alizarin Red S staining (Arrow). 
A

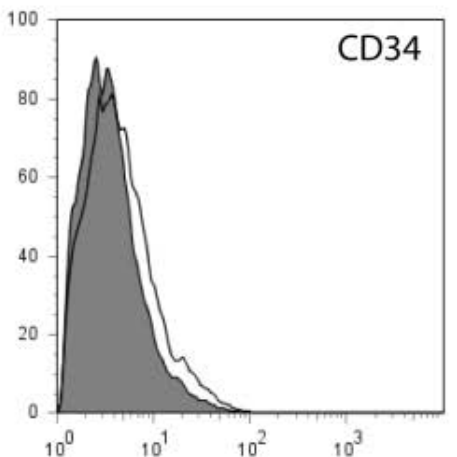

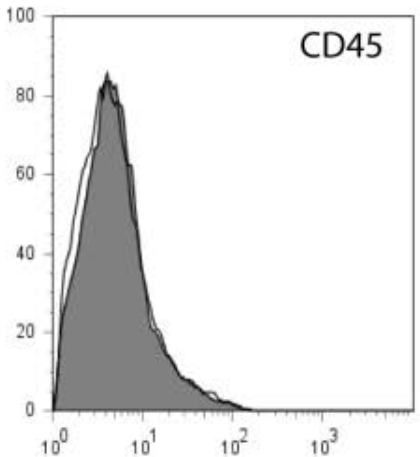
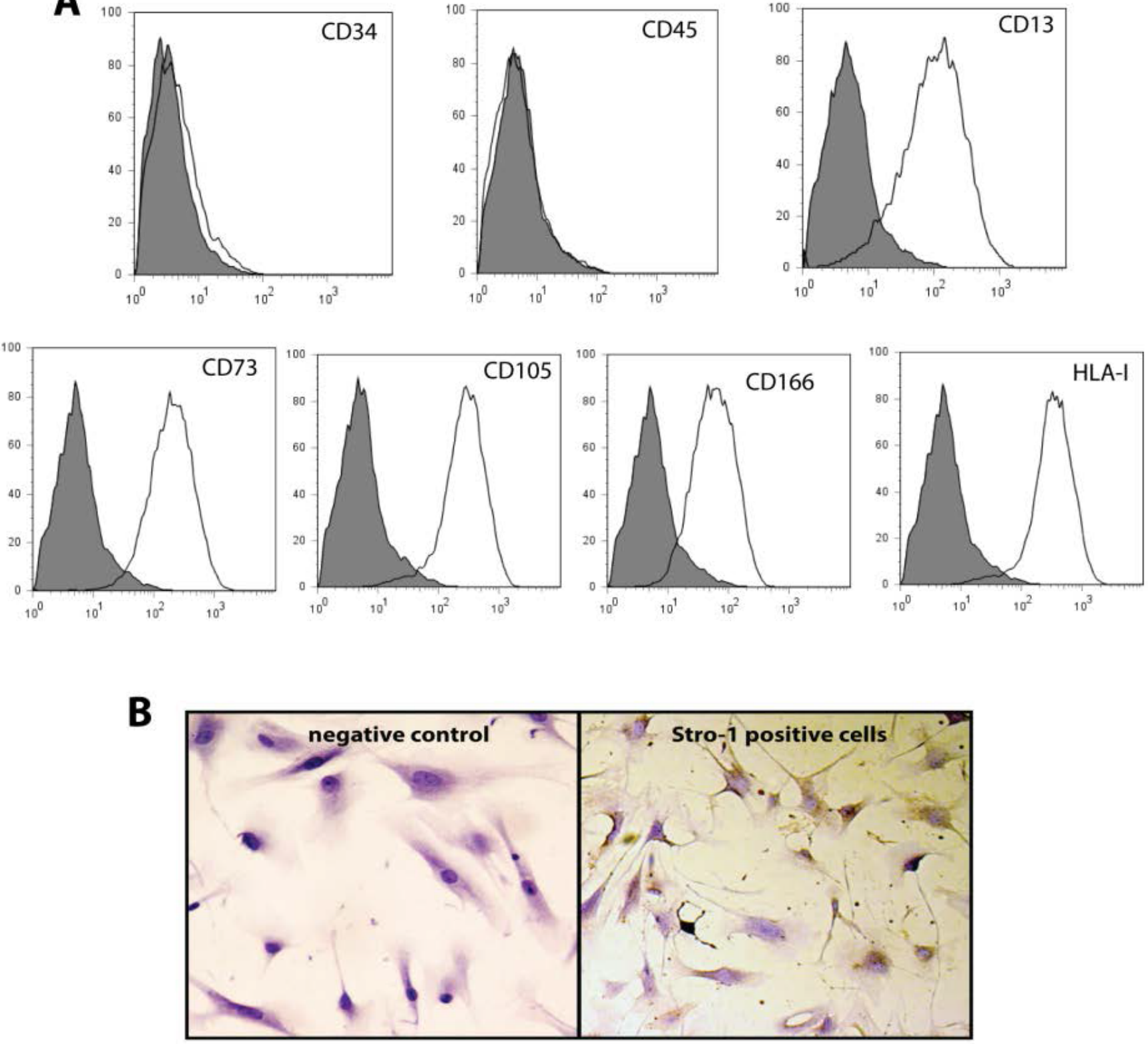

Figure 2: Antigenic profiling of cultured MSCs. (A) FACS staining confirmed that these cells are positive for common mesenchymal markers CD13, CD73, CD105 and CD166 and are negative for the common hematopoietic markers CD34 and CD45. (B) Expression of STRO-1 protein, the best-known MSCs marker, confirmed by immunohistochemical staining of cultured tumor MSCs.

lymph node during a wide-spread tumor metastasis from the colon to an abdominal mass [17]. SW620 cells were seed for colony forming assay and subsequently treated with MSC-conditioned medium (MSC$\mathrm{CM})$. This treatment significantly increased both the number and size of the SW620 colonies $(251.3 \pm 46.9$ vs. $282.1 \pm 55.6$ colonies/ 500 SW620, Mean $\pm \mathrm{SD}, \mathrm{n}=6, \mathrm{P}<0.05)$ and the size $(184.0 \pm 33.6$ vs. $222.8 \pm$ 57.8 pixels, $\mathrm{n}=30, \mathrm{P}<0.01$ ) of $\mathrm{SW} 620$ formed colonies (Figures $4 \mathrm{C}$ and $4 \mathrm{D})$. These results indicate that trophic factors secreted by human liver MSCs provide a potent stimulus to support CRC growth in the liver environment.

\section{Discussion}

CRC tends to metastasize to the liver, which may occur in $20 \%$ to $70 \%$ of patients and represents the major cause of death [19]. The liver holds a distinct cellular and molecular environment [20] and structural changes of tumor microenvironment of CRC often occur once metastasize to the liver [21]. Recent studies have demonstrated the involvement of MSCs as an important cellular element within tumor microenvironments [22].

MSCs were initially discovered in the BM stromal compartment, but lately were identified in number of organs/tissues, including the liver [14]. Extensive studies have demonstrated that MSCs can migrate to the tumor site and incorporate into its microenvironment $[23,24]$. It has also been reported that MSCs migrate to colorectal tumors $[25,26]$. The common approach to study the homing of MSCs into tumor is transplanting culture expanded MSCs into animal models bearing 
Citation: Hernanda PY, Gonzalez AP, van der Laan LJW, Hoogduijn MJ, Peppelenbosch MP, et al. (2013) Mesenchymal Stem/Stromal Cells Exert Trophic Effect on Colorectal Cancer Metastasis to the Liver. J Liver 2: 135. doi:10.4172/2167-0889.1000135

A

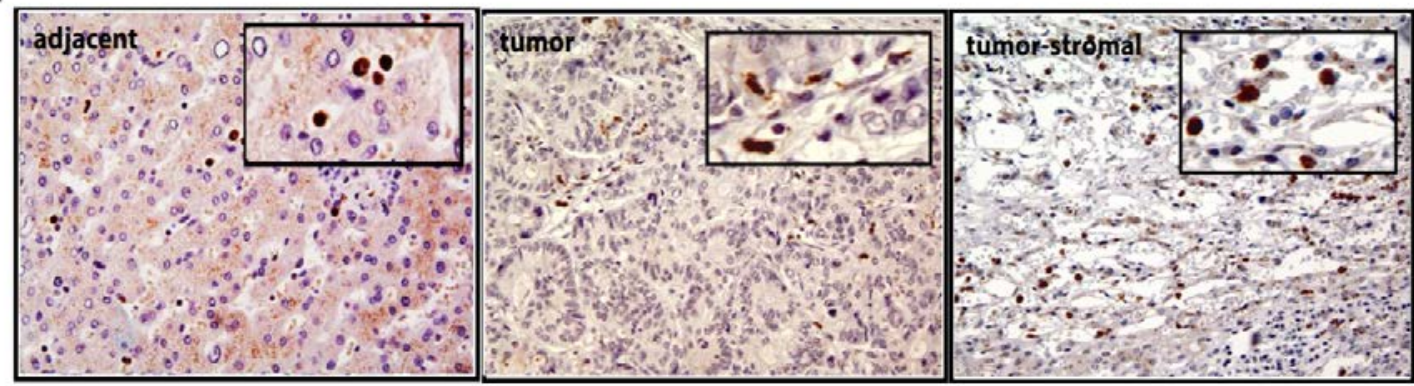

B

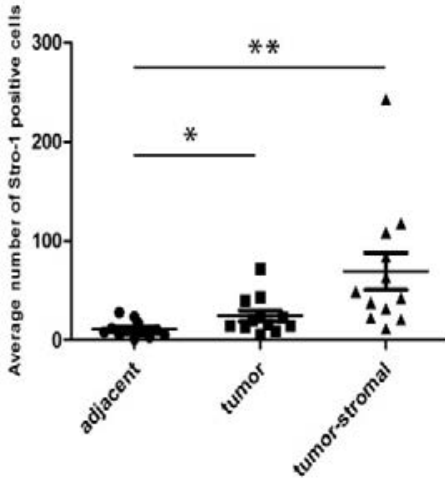

C

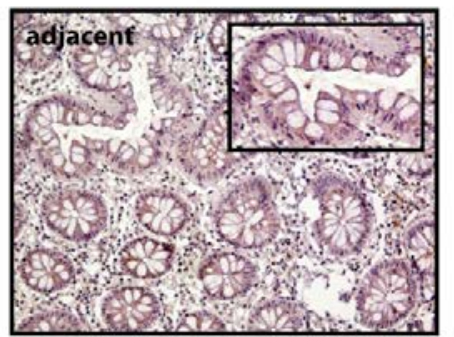

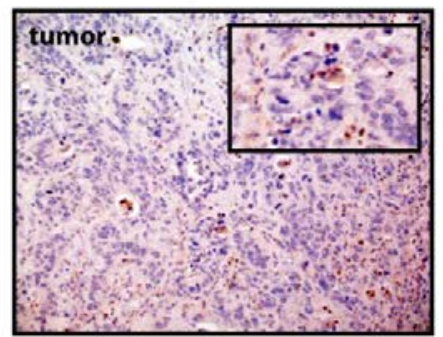

Figure 3: In situ localization of STRO-1 positive cells in paraffin-embedded patient primary CRC and CRC-LM tissues. (A) Distribution of STRO-1 cells in the adjacent, tumor and tumor-stroma regions in CRC-LM tissues. (B) STRO-1 positive cells (an average from 3 HP fields) are significantly enriched in the tumor, in particular the tumor-stroma region, compared with the normal adjacent area in CRC-LM tissues $\left(n=12,{ }^{*} P<0.05\right.$, $\left.{ }^{* *} P<0.01\right)$. (C) In primary CRC tissues, STRO-1 positive cells are present in the tumor but hardly found in the normal adjacent area.

\section{A}

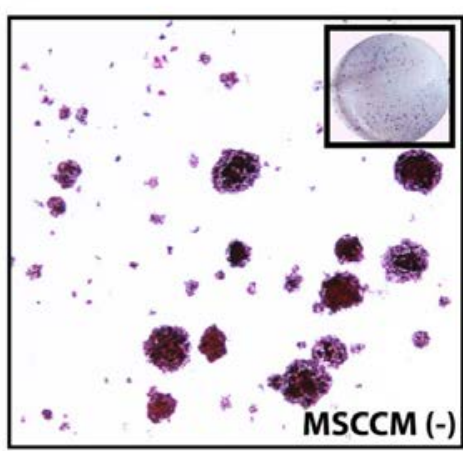

C

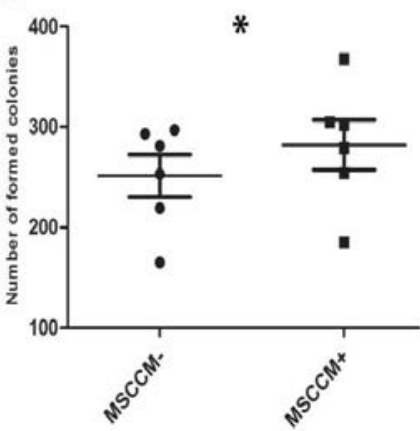

B

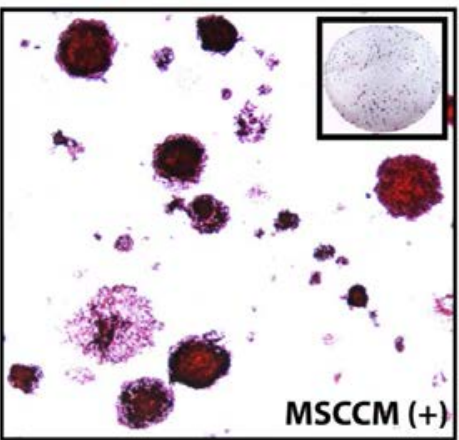

D

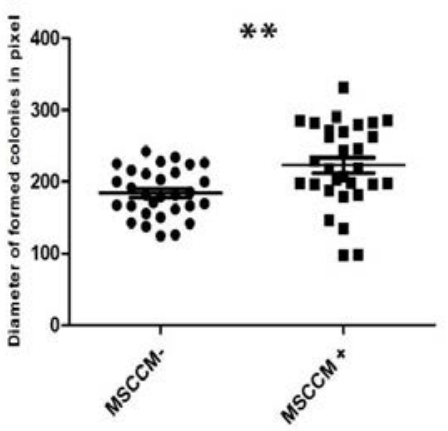

Figure 4: Trophic factors secreted by MSCs promote colony formation and growth of metastasis CRC cells. SW620 treated with MSCs conditioned medium (MSC-CM) resulted in formation of more and larger colonies (A \& B). Treatment of SW620 with MSC conditioned medium significantly increased both the number (251.3 \pm 46.9 Vs $282.1 \pm 55.6$ colonies/ 500 SW620, Mean \pm SD, $n=6, P<0.05)(C)$ and the size $(184.0 \pm 33.6$ Vs $222.8 \pm 57.8$ pixels, $n=30, P<0.01)(D)$ of $S W 620$ formed colonies. 
Citation: Hernanda PY, Gonzalez AP, van der Laan LJW, Hoogduijn MJ, Peppelenbosch MP, et al. (2013) Mesenchymal Stem/Stromal Cells Exert Trophic Effect on Colorectal Cancer Metastasis to the Liver. J Liver 2: 135. doi:10.4172/2167-0889.1000135

Page 5 of 5

tumor [24]. In this study, we have successfully located candidate MSCs in patient CRC-LM and adjacent tissue by immunohistochemical staining with a well-established MSCs Marker, STRO-1. Interestingly, the frequency of STRO-1 cells is significantly higher in the tumor region, in particular the tumor-stromal area of CRC-LM, compared with paired normal adjacent liver tissues. These cells can be cultured from resected CRC-LM tissues and expanded in vitro. This tumorhoming property of MSCs is likely regulated by several specific danger signals and chemotactic factors $[27,28]$, although the exact mechanisms remain elusive.

There is constant debate whether MSCs suppress or support tumor growth and progression [14]. Several studies demonstrated anti-tumor effects [8-10] whereas others showed tumor promoting effects [11-13] of MSCs in different tumor models. Regarding the effects on CRC, most of the studies have shown a pro-cancer effect of MSCs [29-31]. Consistently, we observed that trophic factors secreted by MSCs can promote colony formation and growth of a metastasis CRC cell line. MSCs secrete paracrine factors including a number of growth factors that are known to influence cancer cell proliferation, migration, and angiogenesis [14].

In summary, this study has demonstrated the presence and local selective enrichment of MSCs in human CRC-LM. Trophic factors produced by MSCs can favor the growth of metastasis CRC cells. These results may help to understand the role of MSCs in favoring CRC liver metastasis. In addition, it cautions against the application of anticancer therapy or anti-cancer gene/drug delivery using MSCs as these may intrinsically favor the cancerous process.

\section{Acknowledgments}

Support from the Netherlands Organization for Scientific Research (ZonMw/ NWO), in the form of VENI-grant 916-13-032 to QP and ALW grant 840.12.001 to MP, is gratefully acknowledged. The author are indebted to Dr Liudmila Kodach for providing the SW620 cells and thank Dr. Kostas Sideras Dr. Mirelle E. E. Bröker, Dr. Cornelis Verhoef, Prof. Jan N. M. ljzermans and Dr. Jaap Kwekkeboom for technical support.

\section{References}

1. Siegel R, Ward E, Brawley O, Jemal A (2011) Cancer statistics, 2011: the impact of eliminating socioeconomic and racial disparities on premature cancer deaths. CA Cancer J Clin 61: 212-236.

2. Manfredi S, Bouvier AM, Lepage C, Hatem C, Dancourt V, et al. (2006) Incidence and patterns of recurrence after resection for cure of colonic cancer in a well defined population. Br J Surg 93: 1115-1122.

3. Pfeiffer P, Qvortrup C, Eriksen JG (2007) Current role of antibody therapy in patients with metastatic colorectal cancer. Oncogene 26: 3661-3678.

4. Friedenstein AJ, Deriglasova UF, Kulagina NN, Panasuk AF, Rudakowa SF et al. (1974) Precursors for fibroblasts in different populations of hematopoietic cells as detected by the in vitro colony assay method. Exp Hematol 2: 83-92.

5. Keating A (2012) Mesenchymal stromal cells: new directions. Cell Stem Cell 10: 709-716.

6. Gao Z, Zhang L, Hu J, Sun Y (2012) Mesenchymal stem cells: a potential targeted-delivery vehicle for anti-cancer drug, loaded nanoparticles. Nanomedicine 9: 174-184.

7. Dai LJ, Moniri MR, Zeng ZR, Zhou JX, Rayat J, et al. (2011) Potential implications of mesenchymal stem cells in cancer therapy. Cancer Lett 305: 8-20.

8. Khakoo AY, Pati S, Anderson SA, Reid W, Elshal MF, et al. (2006) Human mesenchymal stem cells exert potent antitumorigenic effects in a model of Kaposi's sarcoma. J Exp Med 203: 1235-1247.

9. Qiao L, Xu Z, Zhao T, Zhao Z, Shi M, et al. (2008) Suppression of tumorigenesis by human mesenchymal stem cells in a hepatoma model. Cell Res 18: $500-$ 507.

10. Zhu Y, Sun Z, Han Q, Liao L, Wang J, et al. (2009) Human mesenchymal stem cells inhibit cancer cell proliferation by secreting DKK-1. Leukemia 23:925-933.
11. Karnoub AE, Dash AB, Vo AP, Sullivan A, Brooks MW, et al. (2007) Mesenchymal stem cells within tumour stroma promote breast cancer metastasis. Nature 449: 557-563.

12. Galie M, Konstantinidou G, Peroni D, Scambi I, Marchini C, et al. (2008) Mesenchymal stem cells share molecular signature with mesenchymal tumor cells and favor early tumor growth in syngeneic mice. Oncogene 27: 25422551.

13. Kucerova L, Matuskova M, Hlubinova K, Altanerova V, Altaner C (2010) Tumor cell behaviour modulation by mesenchymal stromal cells. Mol Cancer 9: 129.

14. Klopp AH, Gupta A, Spaeth E, Andreeff M, Marini F, 3rd (2011) Concise review: Dissecting a discrepancy in the literature: do mesenchymal stem cells support or suppress tumor growth? Stem Cells 29: 11-19.

15. Pan Q, Fouraschen SM, Kaya FS, Verstegen MM, Pescatori M, et al. (2011) Mobilization of hepatic mesenchymal stem cells from human liver grafts. Liver Transpl 17: 596-609.

16. Hernanda PY, Pedroza-Gonzalez A, van der Laan LJ, Broker ME, Hoogduijn MJ, et al. (2013) Tumor promotion through the mesenchymal stem cell compartment in human hepatocellular carcinoma. Carcinogenesis 34: 23302340 .

17. Leibovitz A, Stinson JC, McCombs WB, 3rd, McCoy CE, Mazur KC, et al. (1976) Classification of human colorectal adenocarcinoma cell lines. Cancer Res 36: 4562-4569.

18. Kolf CM, Cho E, Tuan RS (2007) Mesenchymal stromal cells. Biology of adult mesenchymal stem cells: regulation of niche, self-renewal and differentiation. Arthritis Res Ther 9: 204

19. Cunningham D, Findlay M (1993) The chemotherapy of colon cancer can no longer be ignored. Eur J Cancer 29A: 2077-2079.

20. Holz LE, McCaughan GW, Benseler V, Bertolino P, Bowen DG (2008) Liver tolerance and the manipulation of immune outcomes. Inflamm Allergy Drug Targets 7: 6-18.

21. Giusca SE, Carasevici E, Eloae-Zugun F, Tarcoveanu E, Caruntu ID (2008) [Structural changes of tumor microenvironment in liver metastases of colorectal carcinoma] Modificari structurale de micromediu tumoral in metastazele hepatice ale carcinomului colorectal. Rev Med Chir Soc Med Nat lasi 112: 165173.

22. D'Souza N, Burns JS, Grisendi G, Candini O, Veronesi E, et al (2012) MSC and Tumors: Homing, Differentiation and Secretion Influence The Therapeutic Potentials. Adv Biochem Eng Biotechnol.

23. Xia X, Ji T, Chen P, Li X, Fang Y, et al. (2011) Mesenchymal stem cells as carriers and amplifiers in CRAd delivery to tumors. Mol Cancer 10: 134.

24. Lazennec G, Jorgensen C (2008) Concise review: adult multipotent stromal cells and cancer: risk or benefit? Stem Cells 26: 1387-1394.

25. Shinagawa K, Kitadai Y, Tanaka M, Sumida T, Kodama M, et al. (2010) Mesenchymal stem cells enhance growth and metastasis of colon cancer. Int J Cancer 127: 2323-2333.

26. Liu Y, Han ZP, Zhang SS, Jing YY, Bu XX, et al. (2011) Effects of inflammatory factors on mesenchymal stem cells and their role in the promotion of tumor angiogenesis in colon cancer. J Biol Chem 286: 25007-25015.

27. Ringe J, Strassburg S, Neumann K, Endres M, Notter M, et al. (2007) Towards in situ tissue repair: human mesenchymal stem cells express chemokine receptors CXCR1, CXCR2 and CCR2, and migrate upon stimulation with CXCL8 but not CCL2. J Cell Biochem 101: 135-146.

28. Kim WS, Park BS, Sung JH, Yang JM, Park SB, et al. (2007) Wound healing effect of adipose-derived stem cells: a critical role of secretory factors on human dermal fibroblasts. J Dermatol Sci 48: 15-24.

29. De Boeck A, Pauwels $P$, Hensen K, Rummens JL, Westbroek W, et al. (2013) Bone marrow-derived mesenchymal stem cells promote colorectal cancer progression through paracrine neuregulin 1/HER3 signalling. Gut 62: 550-560.

30. Huang WH, Chang MC, Tsai KS, Hung MC, Chen HL, et al. (2012) Mesenchymal stem cells promote growth and angiogenesis of tumors in mice. Oncogene 32 4343-4354.

31. Tsai KS, Yang SH, Lei YP, Tsai CC, Chen HW, et al. (2011) Mesenchymal stem cells promote formation of colorectal tumors in mice. Gastroenterology 141: 1046-1056. 